\title{
ChemComm
}

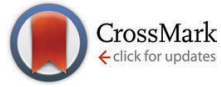

Cite this: Chem. Commun., 2016, 52,4577

Received 5th February 2016, Accepted 29th February 2016

DOI: $10.1039 / c 6 c c 01182 a$

www.rsc.org/chemcomm

\section{Sensitisation of visible and NIR lanthanide emission by InPZnS quantum dots in bi-luminescent hybrids $\uparrow$}

\author{
Jennifer K. Molloy, $\ddagger^{\mathrm{ab}}$ Christophe Lincheneau, $\ddagger^{\mathrm{ac}}$ Maria Moula Karimdjy, $\ddagger^{\mathrm{ab}}$ \\ Fabio Agnese, ${ }^{\text {ac }}$ Lucia Mattera, ${ }^{\text {ac }}$ Christelle Gateau, ${ }^{\text {ab }}$ Peter Reiss, ${ }^{\text {ac }}$ Daniel Imbert*ab \\ and Marinella Mazzanti*d
}

The synthesis of stable hybrid nanoparticles combining InPZnS@ZnSe/ ZnS quantum dots (QDs) and grafted lanthanide complexes has been performed using two different approaches in organic and aqueous media. The final bi-luminescent hybrids exhibit $\mathrm{Ln}^{\mathrm{III}}$ ( $\mathrm{Ln}=\mathrm{Eu}$ and $\mathrm{Yb}$ ) centred luminescence upon QD excitation, suggesting that an energy transfer occurs from the QD to the lanthanide.

The lanthanide ions have attracted increasing attention for a broad range of applications from material science to bioanalysis due to their remarkable intrinsic photophysical properties (narrow emission lines, large effective Stokes shifts, high resistance to photobleaching). ${ }^{1-3}$ Intense luminescence and high stability are crucial for the technological applications of lanthanide complexes in the areas of bioanalysis, energy conversion (luminescent dyes or solar concentrators) or in devices such as light emitting diodes. ${ }^{4,5}$ Low energy excitation is also a requirement for these applications, but due to the low molar absorption coefficient of lanthanide transitions (less than $10 \mathrm{M}^{-1} \mathrm{~cm}^{-1}$ ) it requires the use of suitable chromophores capable of sensitisation of the $\mathrm{Ln}^{\mathrm{III}}$ emission (antenna effect). Moreover high quantum yields are obtained when Ln $^{\text {III }}$ ions are complexed by well-adapted ligands. Most studies have focused on the sensitisation of lanthanide-based luminescence by directly coordinated ligands through the ligand-based triplet excited state, resulting in highly luminescent $\mathrm{Eu}^{\text {III 6-8 }}$ and $\mathrm{Tb}^{\mathrm{III}} 6,9$ complexes. d-block and f-block metal complexes are also increasingly used as effective chromophores to sensitise lanthanide luminescence emission both in the visible and near-IR range. ${ }^{10-13}$

\footnotetext{
${ }^{a}$ Univ. Grenoble Alpes, INAC-SCIB, RICC, F-38000 Grenoble, France

${ }^{b}$ CEA, INAC-SCIB, RICC, F-38000 Grenoble, France

${ }^{c}$ CEA, INAC-SPRAM, LEMOH, F-38000 Grenoble, France

${ }^{d}$ Institut des Sciences et Ingénierie Chimiques, Ecole Polytechnique Fédérale de

Lausanne (EPFL), CH-1015 Lausanne, Switzerland.

E-mail: marinella.mazzanti@epfl.ch

$\dagger$ Electronic supplementary information (ESI) available: Synthetic details for the preparation of ligands and hybrids and spectroscopic characterization. See DOI: 10.1039/c6cc01182a

\$ Contributed equally to this body of work.
}

Förster resonance energy transfer (FRET) from visible emitting $\mathrm{Eu}^{\mathrm{III}}$ and $\mathrm{Tb}^{\mathrm{III}}$ complexes, as energy donors, to CdSe/ZnS quantum dots (QDs), has been reported and Ln-QD hybrids have proven highly sensitive tools in time-resolved fluoro-immunoassays, and multiplexed diagnostics. ${ }^{14-17}$ Moreover, bi-luminescent Ln-QD hybrids with energy transfer from the lanthanide to the QD can also find applications as light sources in white LEDs. ${ }^{18}$

On the other hand, the size-tuneable absorption and emission wavelengths and the large single- and two-photon absorption cross sections make QDs appealing chromophores for the sensitisation of various $\mathrm{Ln}^{\mathrm{III}}$ ions. However, to date only the luminescence sensitisation of $\mathrm{Tb}^{\mathrm{III}}$ in doped CdSe and of Eu ${ }^{\mathrm{III}}$ in doped InPZnS nanocrystals has been reported. ${ }^{19,20}$ The sensitisation of $\mathrm{Ln}^{\mathrm{III}}$ emission by QDs has not been achieved so far in Ln-QD organic/inorganic hybrids. Here, we report the synthesis, physical and chemical characterisation of a new series of bi-luminescent hybrid materials. They are based on InPZnS@ZnSe/ZnS core/shell QDs functionalised with podands or self-assembled lanthanide complexes.

Core/shell InPZnS@ZnSe/ZnS QDs with a hydrodynamic diameter of $\approx 5.5 \mathrm{~nm}$ were synthesised in a two-step procedure building on our earlier work. ${ }^{21} \mathrm{UV}$-vis absorption, luminescence emission, DLS and TEM analyses ( $c f$. ESI $\dagger$ ) are consistent with those reported in the literature. ${ }^{22}$ The QDs used here showed a PL emission maximum in the wavelength range of 525-540 nm and fluorescence quantum yield (QY) of around $40 \%$ in organic solvents (QD-sur, where sur is a mixture of four lipophilic surfactants used during synthesis, see ESI $\dagger$ ). After phase transfer to aqueous solution the QY dropped to around 15\% (QD-pen, where pen refers to penicillamine coating obtained by replacement of the lipophilic surfactants).

Two different $\mathbf{L n}^{\mathrm{III}}$ complexes 1-Ln and 2-Ln were grafted onto the QDs according to the procedure shown in Scheme 1. The complexes 1-Ln ( $\mathrm{Ln}=\mathrm{Eu}, \mathrm{Tb}, \mathrm{Gd}$ and $\mathrm{Yb})$ were prepared using a multi-step synthesis (ESI, $\dagger$ Scheme S1 and Fig. S1). High thermodynamic stability in water was reported for the closely related $[\mathrm{Gd}(\mathrm{ebpatcn})]$ complex and similar stability with respect to ligand dissociation is expected for the complexes 1-Ln. ${ }^{23}$ The complexes 1-Eu ${ }^{\text {III }}$ and $\mathbf{1 - T} \mathbf{T b}^{\text {III }}$ show significant luminescence 


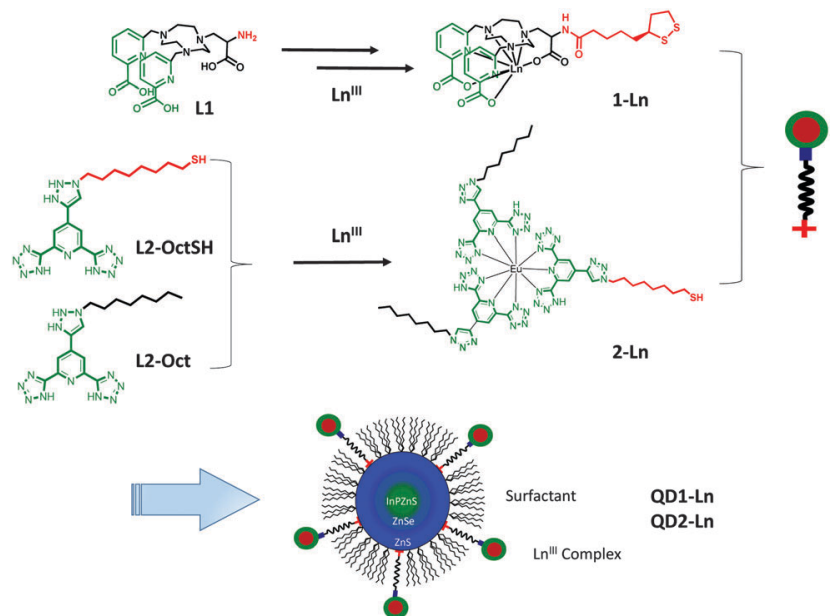

Scheme 1 Synthesis of the lanthanide complexes 1-Ln and 2-Ln and of the hybrids QD1-Ln and QD2-Ln ( $\mathrm{Ln}=\mathrm{Eu}, \mathrm{Tb}, \mathrm{Gd}$ and $\mathrm{Yb})$.

quantum yields, (10 and 19\% in water, 22 and $40 \%$ in $\mathrm{D}_{2} \mathrm{O}$ for $\mathrm{Eu}^{\mathrm{III}}$ and $\mathrm{Tb}^{\mathrm{III}}$, respectively). L2-OctSH and L2-Oct were synthesised from the chelidamic acid following the procedure developed by Di Pietro et al. ${ }^{6}$ for L2-Oct and a modified procedure for L2-OctSH (Scheme S2, ESI $\dagger$ ). Previous work suggested that the close proximity of the Ln complex to the QD surface can facilitate photoinduced electron transfer from the QDs to the complex resulting in the quenching of QD emission. ${ }^{24}$ Various spacer lengths (3 to 12 carbons)§ were therefore investigated and a chain of eight carbon atoms was found to be optimum. It leads to a high number of grafted complexes while being short enough to enable QD-to-Ln energy transfer processes, and long enough to favour them over electron transfer. The L2-Oct ligands form homoleptic (L2-Oct) $)_{3}-\mathbf{L n}$ complexes that are highly stable $\left(\log \beta_{13} \approx 19\right.$ in methanol). ${ }^{25}$ Similar results were observed with $(\mathbf{L 2}-\mathbf{O c t S H})_{3}-\mathbf{L n}\left(\log \beta_{13} \approx 17\right.$ in methanol and $\log \beta_{13} \approx 18.2$ in $\mathrm{CHCl}_{3}$ ) and analogous stability is expected for 2-Ln (Fig. S2, ESI $\dagger$ ). The (L2-oct) ${ }_{3}$ - $\mathbf{L n}$ complexes showed high luminescence quantum yields in the case of $\mathrm{Eu}^{\text {III }}$ (70\%) and $\mathrm{Tb}(96 \%)$ and a measurable emission for $\mathrm{Yb}^{\mathrm{III}}{ }^{6}$

The hybrids QD1-Ln ( $\mathbf{L n}=\mathrm{Eu}, \mathrm{Tb}, \mathrm{Yb})$ were synthesised following a previously reported procedure ${ }^{26}$ via the post functionalisation of QD-pen by the $\operatorname{Ln}^{\text {III }}$ complexes 1-Ln in the presence of a reducing agent. QD1-Ln $(\mathrm{Ln}=\mathrm{Eu}, \mathrm{Tb}, \mathrm{Yb}$ and Gd) were obtained as pure hybrids after size exclusion column chromatography. The number of complexes per QD (115) was determined by combining two techniques, absorption spectroscopy (QD concentration) and magnetic susceptibility (complex concentration). DLS measurements revealed that the QD1-Ln hybrids show a small hydrodynamic diameter of $\sim 9.5 \mathrm{~nm}$ and narrow size distribution with polydispersity indices of an average of 0.4 (Fig. 1 and Fig. S4, Table S1, ESI $\dagger$ ). QD-pen exhibits a hydrodynamic diameter, which is $1.5 \mathrm{~nm}$ larger than the TEM diameter, corresponding to a $0.75 \mathrm{~nm}$ thick ligand shell in accordance with the compact pen-capping. Moreover, the increase of the hydrodynamic diameter of approximately $4 \mathrm{~nm}$ from QD-pen to QD1-Ln is consistent with the successful grafting of the complexes at the QD surface. The formation of the hybrids QD2-Ln was
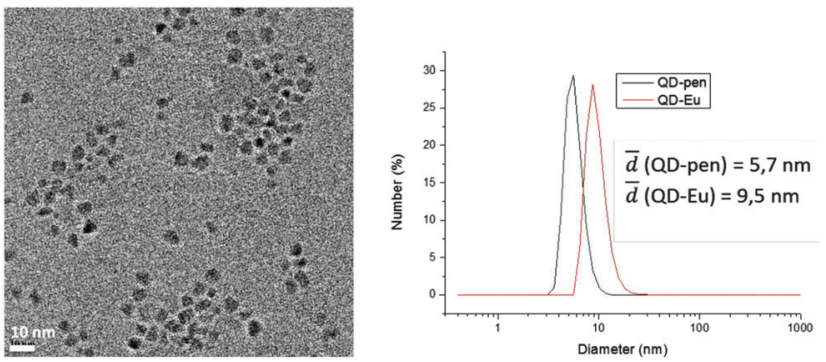

Fig. 1 Left: TEM micrographs of QD-pen (mean size: $4.2 \pm 0.5 \mathrm{~nm}$ ). Right: dynamic light scattering data and hydrodynamic diameters of QD-pen and QD1-Eu.

performed using a novel three-step strategy relying on the welldefined coordination chemistry of the L2-Oct ligands enabling the controlled supramolecular build-up of the hybrids. It involves purification by extraction and characterisation by absorption and emission spectroscopy at each stage (ESI $\dagger$ ). The first step involved a partial exchange in chloroform of the surfactants in QD-sur (TEM, Fig. S5, ESI $\dagger$ ) with L2-OctSH to afford the hybrids QD-L2-OctSH (Fig. S6, ESI $\dagger$ ). Reaction of QD-L2-OctSH with 1 eq. of $\operatorname{Ln}(\mathrm{OTf})_{3}$ $(\mathrm{Ln}=\mathrm{Eu}, \mathrm{Tb}, \mathrm{Yb})$ per grafted ligand (QD-L2-OctSH·Eu, Fig. S7, ESI $\dagger$ ), followed by the addition of 2 eq. of L2-Oct per metal ion afforded the hybrids QD2-Ln (Fig. S8, ESI $\dagger$ ). Absorption spectroscopy indicates the presence of 200 grafted complexes per QD.

The photophysical properties of QD1-Ln (Fig. 2 and Fig. S9-S11, ESI $\dagger$ ) and QD2-Ln (Fig. 3) were then analysed for $\mathbf{L n}=\mathrm{Eu}$, Tb and $\mathrm{Yb}$. After grafting of the ligands or complexes on the surface, the emission QY of the QDs shows a quenching from 40 and $15 \%$ for QD-sur and QD-pen to 5-40\% and 1-15\%, respectively. Direct excitation of QD1-Ln at $273 \mathrm{~nm}$ (corresponding to the absorption band of the ligand $\mathbf{L 1}$ but also to the absorption of the QD) resulted in the characteristic $\operatorname{Eu}\left({ }^{5} \mathrm{D}_{0} \rightarrow{ }^{7} \mathrm{~F}_{j}\right)$ and $\mathrm{Tb}\left({ }^{5} \mathrm{D}_{4} \rightarrow{ }^{7} \mathrm{~F}_{j}\right)$ luminescence emission of the $\mathrm{Eu}^{\mathrm{III}}$ and $\mathrm{Tb}^{\mathrm{III}}$ complexes (Fig. 2, left and ESI $\dagger$ ) through ligand excitation. In addition the emission spectra show a band centred at $525 \mathrm{~nm}$ resulting from excitation of the QDs.

The excitation at $273 \mathrm{~nm}$, through the absorption band of the ligand, or at $370 \mathrm{~nm}$ of the free $\mathrm{Yb}^{\mathrm{III}}$ complex did not lead to emission in the NIR region. Notably, the presence of a coordinated water molecule leads to the deactivation of the $\mathrm{Yb}^{\mathrm{III}}$ centred luminescence. However, the free $\mathrm{Eu}^{\mathrm{III}}$ complex shows a sizeable
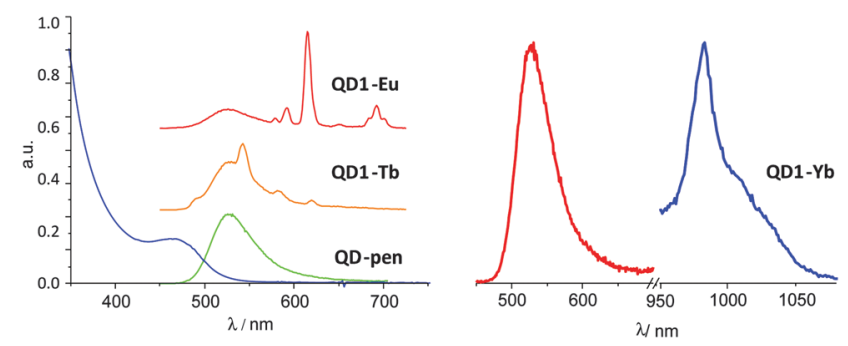

Fig. 2 (left) Normalised absorption of QD-pen (blue) and emission $\left(\lambda_{\text {ex }}=\right.$ $273 \mathrm{~nm}$ ) spectra of QD-pen (green), QD1.Ln (Tb: orange, Eu: red); (right) normalised emission spectra, $\left(\lambda_{\mathrm{ex}}=370 \mathrm{~nm}\right)$ of QD1-Yb in the Vis (red) and NIR range (blue). All measurements were performed in PBS $1 \times$ buffer. 


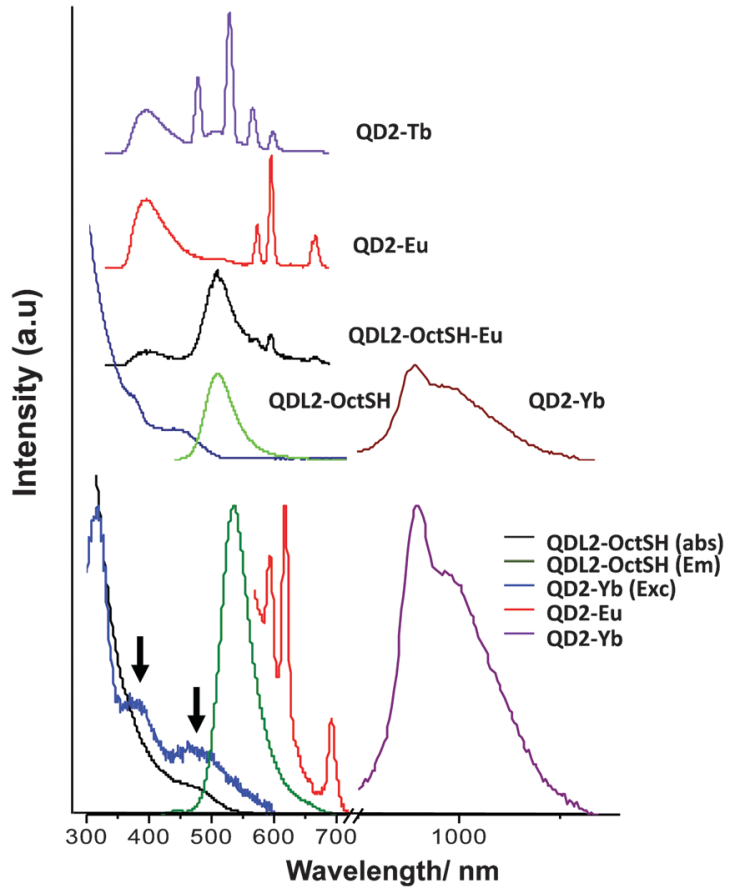

Fig. 3 Photophysical characterisation of QD2- $\mathbf{L n}\left(4 \times 10^{-8} \mathrm{M}\right)$ in $\mathrm{CHCl}_{3}$ (top) normalised absorption spectrum of QD-L2-OctSH (blue) and emission spectra ( $\lambda_{\mathrm{ex}}=325 \mathrm{~nm}$ ) of QD-L2-OctSH (green), QD-L2-OctSH.Eu (black) and QD2.Ln (Tb: violet, Eu: red, Yb: brown); (bottom) normalised absorption spectrum of QD-L2-OctSH (black) and emission spectra ( $\lambda_{\text {ex }}=$ $400 \mathrm{~nm}$ ) of QD-L2-OctSH (green) and QD2. Ln (Eu: red, Yb: violet). In blue, the excitation spectrum of QD2.Yb $\left(\lambda_{\text {an }}=978 \mathrm{~nm}\right)$.

luminescence upon excitation through the ligand at $273 \mathrm{~nm}$ but no emission upon excitation at $370 \mathrm{~nm}$ (no residual ligand absorption).

When grafted onto QD-pen, excitation at 273 (excitation of both ligand and QD) and $370 \mathrm{~nm}$ (excitation of QD) of QD1·Eu results in a strong and very weak emission of $\mathrm{Eu}^{\mathrm{III}}$ at $618 \mathrm{~nm}$, respectively. For QD1·Yb, excitation at $370 \mathrm{~nm}$ gives a weak luminescence emission despite the presence of the coordinated water molecule. The emission spectrum reveals a band at $978 \mathrm{~nm}$ (Fig. 2, right), assigned to the ${ }^{2} \mathrm{~F}_{5 / 2} \rightarrow{ }^{2} \mathrm{~F}_{7 / 2}$ transition and broader vibronic components at longer wavelengths. Excitation at $273 \mathrm{~nm}$ of QD1.Yb results in the absence of emission, probably due to a weaker efficiency of both the Xenon lamp (low power) and the gratings (tail) in this region compared to $370 \mathrm{~nm}$. The combination of these two factors makes the $\mathrm{Yb}^{\mathrm{III}}$ NIR luminescence too weak to be detected at $273 \mathrm{~nm}$ as observed for QD1.Eu. The luminescence emission of the lanthanide ions unambiguously arises via excitation of the QDs as no ligand absorption appears at $\lambda>320 \mathrm{~nm}$. The presence of the QD-sensitised $\mathrm{Eu}^{\mathrm{III}}$ and $\mathrm{Yb}^{\mathrm{III}}$ emission was confirmed by the absence of luminescence when the free complexes 1-Ln were excited at $370 \mathrm{~nm}$.

Moreover, when the $\mathrm{Gd}^{\mathrm{III}}$ ion was replaced by $\mathrm{Yb}^{\mathrm{III}}$ to form QD1·Yb, the QD lifetimes decreased (3, 16, and $62 \mathrm{ns)} \mathrm{com-}$ pared to the values $(4,23$ and $84 \mathrm{~ns}$ ) measured for QD-pen or QD1-Gd, suggesting the presence of an energy transfer from the
QD to the lanthanide ion, associated with a decrease of the QD lifetimes (Table S2, ESI $\dagger$ ). For QD1-Eu, the decrease of the QD lifetimes is more pronounced (measured values of 1, 6 and $30 \mathrm{~ns}$ ) while the $\mathrm{Eu}^{\mathrm{III}}$ emission lifetime measured in the hybrid QD1-Eu is similar to the one measured for the free complex in water ( 0.49 and $0.48 \mathrm{~ms}$, respectively). These data indicate that an energy transfer with additional deactivation pathways occurs in QD1-Eu from QD to $\mathrm{Eu}^{\mathrm{III}}$. Thus, only a residual emission from the $\operatorname{Eu}\left({ }^{5} \mathrm{D}_{0}\right)$ level is observed, the emission of the QD and the first accepting $\operatorname{Eu}\left({ }^{5} \mathrm{D}_{0}\right)$ electronic level being very close in energy (Fig. S9, ESI $\dagger$ ). For QD1-Tb (Fig. S10, ESI $\dagger$ ), the decrease of the QD lifetimes is significantly less important (3, 19, $72 \mathrm{~ns})$ compared to QD1-Yb and QD1-Eu, so only a small energy transfer might occur, if possible at all. In this case, the emission of the QD overlaps the $\operatorname{Tb}\left({ }^{5} \mathrm{D}_{4}\right)$ first accepting level with the presence of a back transfer from the $\mathrm{Tb}\left({ }^{5} \mathrm{D}_{4}\right)$ to the QD $\left(\mathrm{Tb}^{\mathrm{III}}\right.$ lifetimes $=0.66$ and $0.92 \mathrm{~ms}$ for QD1-Tb and 1-Tb, respectively, in aqueous buffer). The nullification of these two energetic pathways could explain the absence of luminescence of $\mathrm{Tb}^{\mathrm{III}}$ in QD1-Tb. Furthermore, the values of the QDs quantum yields measured for the hybrids QD1-Ln, (1, 5, 10, and 15\% for QD1-Eu, -Yb, - Tb and -Gd, respectively, Table S2, ESI $\dagger$ ) follow a similar trend to the observed decrease of the lifetimes. This trend matches the decrease of both lifetimes and emission of the donor usually associated to an energy transfer.

In the case of QD2-L2-OctSH the grafting of the ligands on the surface of the QDs was concomitant with a significant quenching of the QD luminescence emission (decreasing the intensity between 20 and $80 \%$ in comparison with a control sample) depending on the quantity of ligand involved in the functionalisation (Fig. 3). The measurements of the QD emission lifetimes (Table S2, ESI $\dagger$ ) show a decrease from QD-sur (0.3, 17 and $75 \mathrm{~ns})$ to QD-L2-OctSH (0.2, 5 and $50 \mathrm{~ns}$ ). The formation of the complex QD-L2-OctSH-Eu leads to a weak luminescence of $\mathrm{Eu}^{\mathrm{III}}$, whereas completion of the coordination sphere with L2-Oct affording QD2-Eu leads to luminescence emission similar to that found for the free $\left[\mathrm{Eu}(\mathbf{L 2}-\mathbf{O c t})_{2}(\mathbf{L 2}-\mathbf{O c t S H})\right]$ complex and lifetimes for the QD of $0.2,4$ and $31 \mathrm{~ns}$. This suggests that the $\mathrm{Eu}^{\mathrm{III}}$ coordination environment remains similar to that of the free complex upon grafting at the surface

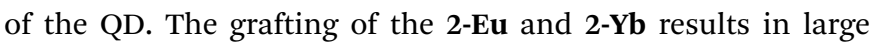
quenching of the QD emission suggesting that a partial energy transfer occurs, while no quenching is observed for 2-Tb $40 \%$ QY for QD2-Tb and QD-sur). Excitation of QD2-Tb at $325 \mathrm{~nm}$, (Fig. 3, top), i.e. on both the transitions of the ligand and the QD, results in the emission of both the QD and the lanthanide ions. When excitation of QD2-Tb was performed at $400 \mathrm{~nm}$ (where only the QD is excited), the emission of the metal was not observed. This could be due to the $\operatorname{Tb}\left({ }^{5} D_{4}\right)$ energy level being too low for an energy transfer or more probably a backtransfer from the $\operatorname{Tb}\left({ }^{5} \mathrm{D}_{4}\right)$ to the QD level as observed in the case of QD1-Tb. For QD1-Ln and QD2-Ln an energy transfer may not be possible by the resonant transfer mechanism due to a lack of absorption/emission overlap. However, phonon-assisted transfer involving energy mismatch and creation and/or annihilation of phonons could occur. 
Conversely, the excitation at low energy (400 nm) of QD2-Eu and QD2-Yb, gave rise to a weak $\mathrm{Eu}^{\text {III }}$ and a sizeable $\mathrm{Yb}^{\text {III }}$ luminescence (Fig. 3, bottom). In the $\mathrm{Yb}^{\mathrm{III}}$ emission spectrum we observe generally one signal in the NIR at approx. $978 \mathrm{~nm}$ and a broad component centred at $1040 \mathrm{~nm}$. This is due to an absorption/reemission of the excitation energy by the $\mathrm{Yb}^{\mathrm{III}}$ energy levels. In addition, the free complexes [Eu(L2-Oct) $)_{2}(\mathbf{L 2}$-OctSH)] and $\left[\mathbf{Y b}(\mathbf{L 2} \text {-Oct })_{\mathbf{2}}\right.$ (L2-OctSH)] did not show any measurable luminescence emission when excited at $400 \mathrm{~nm}$, indicating that the excitation through the ligand cannot occur at this wavelength. Moreover, the strong luminescence emission of the $\mathrm{Yb}^{\mathrm{III}}$ in QD2-Yb allowed the measurement of a well-defined excitation spectrum. Thus, the presence of an energy transfer from the QD to the $\mathrm{Yb}^{\mathrm{III}}$ centre is confirmed by the apparition of two bands at 380 and $450 \mathrm{~nm}$ in the excitation spectrum (matching the low-energy band of the absorption spectrum), corresponding to the sensitisation of the complex via the QD. This result confirms that the successful population of the lanthanide energy levels was achieved via sensitisation of the QD core.

Two new types of QD-Ln ${ }^{\text {III }}$ organic/inorganic hybrids showing dual luminescence have been prepared and characterised using two different approaches. The QDs and $\mathrm{Ln}^{\mathrm{III}}$ complexes have been chosen to promote both dual luminescence and energy transfer. A novel self-assembly strategy provides a straightforward and synthetically undemanding route to the synthesis of QD2-Ln hybrids that are soluble and stable in organic solutions. These hybrids are potentially useful for the development of new luminescent materials. The use of functionalised, water-stable lanthanide complexes gave access to QD1-Ln hybrids with potential application in biological imaging. Overall, the obtained QD-Ln ${ }^{\text {III }}$ hybrids have allowed the demonstration of the potential of using QDs to sensitise both the visible and NIR emission of lanthanide ions incorporated into QD-grafted complexes, either in organic or aqueous media. Future studies will be directed towards the optimisation of the transfer through an appropriate matching of the Ln and QD energy levels and the thorough investigation of the transfer mechanisms.

The authors gratefully acknowledge financial support from the "French Agence Nationale de la Recherche", grant NIRA (ANR-13-BS08-0011), Labex Arcane, ANR-11-LABX-003-01 and the Ecole Polytechnique Fédérale de Lausanne (EPFL). They thank Lydia Plassais and Sebastiano Di Pietro for the synthesis of L1 and L2-Oct.

\section{Notes and references}

$\S$ Unpublished work: The $\mathrm{C}_{3}$ spacer was not suitable for the formation of the self-assembly due to the sterically encumbered environment around QD-sur. The C12 ligand demonstrates a decrease of the lanthanide sensitisation with respect to the $\mathrm{C} 8$ spacer, which was therefore chosen for all further studies.

1 J.-C. G. Bünzli, Coord. Chem. Rev., 2015, 293, 19-47.

2 J.-C. G. Bünzli, Acc. Chem. Res., 2006, 39, 53-61.

3 S. Faulkner, S. J. A. Pope and B. P. Burton-Pye, Appl. Spectrosc. Rev., 2005, 40, 1-31.

4 K. Binnemans, Chem. Rev., 2009, 109, 4283-4374.

5 S. V. Eliseeva and J.-C. G. Bünzli, Chem. Soc. Rev., 2010, 39, 189-227.

6 S. Di Pietro, D. Imbert and M. Mazzanti, Chem. Commun., 2014, 50, 10323-10326.

7 G. Nocton, A. Nonat, C. Gateau and M. Mazzanti, Helv. Chim. Acta, 2009, 92, 2257-2273.

8 M. Delbianco, V. Sadovnikova, E. Bourrier, G. Mathis, L. Lamarque, J. M. Zwier and D. Parker, Angew. Chem., Int. Ed., 2014, 53, 10718-10722.

9 J. Xu, T. M. Corneillie, E. G. Moore, G.-L. Law, N. G. Butlin and K. N. Raymond, J. Am. Chem. Soc., 2011, 133, 19900-19910.

10 T. J. Sørensen, A. M. Kenwright and S. Faulkner, Chem. Sci., 2015, 6, 2054-2059.

11 S. J. A. Pope, B. J. Coe, S. Faulkner, E. V. Bichenkova, X. Yu and K. T. Douglas, J. Am. Chem. Soc., 2004, 126, 9490-9491.

12 S. Faulkner and S. J. A. Pope, J. Am. Chem. Soc., 2003, 125, 10526-10527.

13 D. Imbert, M. Cantuel, J.-C. G. Bünzli, G. Bernardinelli and C. Piguet, J. Am. Chem. Soc., 2003, 125, 15698-15699.

14 D. Geißler, L. J. Charbonnière, R. F. Ziessel, N. G. Butlin, H.-G. Löehmannsröeben and N. Hildebrandt, Angew. Chem., Int. Ed., 2010, 49, 1396-1401.

15 D. Geißler, S. Linden, K. Liermann, K. D. Wegner, L. J. Charbonnière and N. Hildebrandt, Inorg. Chem., 2014, 53, 1824-1838.

16 N. Hildebrandt, K. D. Wegner and W. R. Algar, Coord. Chem. Rev., 2014, 273, 125-138.

17 L. J. Charbonnière and N. Hildebrandt, Eur. J. Inorg. Chem., 2008, 3241-3251.

18 B.-H. Kwon, H. S. Jang, H. S. Yoo, S. W. Kim, D. S. Kang, S. Maeng, D. S. Jang, H. Kim and D. Y. Jeon, J. Mater. Chem., 2011, 21, 12812-12818.

19 D. A. Chengelis, A. M. Yingling, P. D. Badger, C. M. Shade and S. Petoud, J. Am. Chem. Soc., 2005, 127, 16752-16753.

20 U. T. D. Thuy, A. Maurice, N. Q. Liem and P. Reiss, Dalton Trans., 2013, 42, 12606-12610.

21 L. Li, M. Protière and P. Reiss, Chem. Mater., 2008, 20, 2621-2623.

22 U. T. D. Thuy, P. Reiss and N. Q. Liem, Appl. Phys. Lett., 2010, 97, 193104.

23 A. Nonat, M. Giraud, C. Gateau, P. H. Fries, L. Helm and M. Mazzanti, Dalton Trans., 2009, 8033-8046.

24 S. A. Gallagher, S. Comby, M. Wojdyla, T. Gunnlaugsson, J. M. Kelly, Y. K. Gun'ko, I. P. Clark, G. M. Greetham, M. Towrie and S. J. Quinn, Inorg. Chem., 2013, 52, 4133-4135.

25 S. Di Pietro, N. Gauthier, D. Imbert, J. Pécaut and M. Mazzanti, Dalton Trans., 2016, 45, 3429-3442.

26 G. J. Stasiuk, S. Tamang, D. Imbert, C. Poillot, M. Giardiello, C. Tisseyre, E. L. Barbier, P. H. Fries, M. de Waard, P. Reiss and M. Mazzanti, ACS Nano, 2011, 5, 8193-8201. 